\title{
Glass ornament production and trade polities in the Upper-Thai Peninsula during the Early Iron Age
}

\author{
Laure Dussubieux, Bérénice Bellina
}

\section{Introduction}

The Thai-Malay peninsula is at the intersection of the Bay of Bengal (Gupta, 2005; Bellina, in press a) and the South China Sea Interaction Spheres (Solheim, 2006; Hung et al., 2013; Blench, in press; Bellina, in press a). This unique geographical position partly accounts for the peculiar hybrid cultural developments that the upper part of the region witnesses as early as the mid-first millennium as reflected in the urban traits and industries of Khao Sam Kaeo (Bellina, in press a, 2017). As in the case of hard stones (Bellina, 2007, 2014) and possibly of copperbased industries (Pryce et al., 2017), the sudden appearance of complex skills necessary for the production of glass ornaments at Khao Sam Kaeo suggests a transfer of artisans, likely from India. Again, as for siliceous stones which were most likely imported from India (Carter and Dussubieux, 2016), raw glass found at Khao Sam Khao was imported from India through the Bay of Bengal Interaction Sphere to be locally transformed into ornaments with a style shared by communities of the South China Sea Interaction Sphere within which they were then "distributed" as far as the Philippines (Dussubieux and Bellina, 2017).

The excavation of the contemporaneous port-settlement of Khao Sek located $80 \mathrm{~km}$ south of Khao Sam Kaeo and at the end of a river system which is part of major ancient trans-peninsular route and the technological reconstruction and comparison of both sites' industries unraveled striking similarities between the two sites. Those are interpreted as the result of related, ranked and complementary ports-oftrade forming part of a confederation controlling the transpeninsular routes. Khao Sek may have acted as an outpost controlling the flux of the Langsuan river system to redistribute the goods to Khao Sam Kaeo acting as the international market place where foreign traders and artisans stayed (Bellina, this issue a,b). The settlement shows many similarities with that of Khao Sam Kaeo in terms of topography, modes of construction, internal organization of craft activities. At the same time, Khao Sek appears smaller and lacking monumental works evidenced at Khao Sam Kaeo such as the encircling walls, the monumental terraces, drainage and hydraulic system. Khao Sek does not appear as socially complex as Khao Sam Kaeo: it did not yield evidence for the presence of foreigners established at the site within socio-professional compounds such as at Khao Sam Kaeo. The smaller port did not provide imports such as Han materials or Indian Fine Ware for instance although Indian Fine ware and Dong Son drums are found in several transshipment and collecting sites along the River Langsuan system/transpeninsular route such as at Pangwan, Ban Na Hyan and wat Pathumtaram (see Map 2 in 
Bellina, this issue a). Exogenous traits appear in the shape of Indianinfluenced ceramics such as kendi that A. Favereau interprets as produces made at Khao Sam Kaeo and exported to Khao Sek (Favereau, this issue). Khao Sek yielded fragments of one Don Son drum which turned up to be an ancient fake (Pryce and Bellina, this issue) as opposed to Khao Sam Kaeo which yielded several drums of various sizes. Finally, Khao Sam Kaeo furnished more than twenty seals (blanks or inscribed with brahmi inscriptions) whilst only two are reported at Khao Sek, and one of which containing a writing mistake showing the lack of expertise of the artisan. Despite these differences, the fact that the populations in both sites are related is shown by the production of the same domestic ceramics (the two technological groups are found and in similar proportions).

These two sites show evidence for hybrid industries producing similar objects of the "Late Prehistoric South China Sea style" that is found in various sites along the coast of the South China Sea (Bellina, in press a, 2017) and their "chaîne opératoire techniques" is the same (see Bellina, this issue a). This is the case for the glass industry too which is the focus of this study. Like Khao Sam Kaeo, Khao Sek yielded an impressive quantity of comparable glass material including glass wastes suggesting the presence of an active glass workshop at this site. The study of this material presented here includes a general description of the type of artifacts and compositional analysis obtained using laser ablation - inductively coupled plasma - mass spectrometry (LA-ICPMS) and a comparison with that of Khao Sam Kaeo. This study provides data on the elaboration of a regional craft system and how it contributes to what can be labeled a "South China Sea style" (Bellina, in press a) in the context of emergent maritime silk roads trading polities. More broadly, along other analysis of these port-cities industries, this study of the early glass shed light on aspects of the still poorly known emergent maritime polities in Southeast Asia.

\section{Site and glass artifacts}

\subsection{The glass material at Khao Sek}

Khao Sek is an industrial port situated at the mouth of the River Langsuan that emerged during the 4th c. BCE (Fig. 1). It was excavated by the Thai-French Mission in 2013 and 2014; however, most of the glass material was found along a small branch of the river, on the western part of the site which was excavated by the land's owner. Consequently very little contextual information is available for most of the artifacts discussed below and it has not been possible to catalogue all the artifacts individually. Despite these caveats, we are able to provide a general description of the glass corpus found at Khao Sek and to place it in a chronological framework thanks to the small quantity of glass beads and bracelets fragments that were found in-situ by the French-Thai mission and the data obtained through compositional analysis. The material is now kept at the National Museum of Chumphon.

Although we were unable to determine precisely the quantity of glass found at Khao Sek, it is safe to say that several thousand glass artifacts were recovered at the site. A large number of unshaped glass fragments were collected; most of them present evidence of knapping. Unshaped glass fragments with evidence of hot working are available too but in much smaller number. Other glass artifact types recovered at the site includes: refuses of bracelet manufacturing, finished bracelets and beads (Fig. 2).

Pieces of unshaped glass or raw glass are turquoise blue, green, black, red and dark blue. There are two shades of turquoise blue, one being of a more intense color than the other one. The turquoise blue glass is translucent, the green and the dark blue glasses are transparent and the red glass is opaque. In a sample of 162 pieces of unshaped glass artifacts, the proportion of the turquoise blue pieces is $47.5 \%$, transparent green, $44 \%$ and black $2.4 \%$. Red glass and dark blue glass represent each $1.8 \%$ of the total. The color of a few artifacts could not be identified.

Glass beads were manufactured by the drawn, the wound or the lapidary techniques. Drawn beads are mostly opaque red and transparent dark blue. They are by far the most abundant type of beads at the site. No evidence of drawn bead manufacturing is available as no tubular pieces of glass were visible and red and dark blue waste glass fragments were not very abundant. Based on the lack of local manufacturing evidence, drawn beads might have been imported from elsewhere.

A fairly large number of lapidary glass beads, estimated to more than a hundred, were recovered at Khao Sek. They are transparent green, translucent dark blue, colorless or amber and are available in a variety of shapes. Broken or whole specimens are visible. It is impossible to say whether the broken specimens were production wastes or broken after they were manufactured. A local production is possible as testified by the large quantities of knapped wastes that could have been generated by lapidary glass bead manufacturing. Pelegrin (2000), a specialist in stone-knapping technologies was able to identify flake scars on knapped waste from Khao Sam Khaeo characterizing indirect percussion by the counter-blow technique, a traditional Indian technique also called the "Cambay technique", used since the Harappan period. This technique was used within the same workshop to work siliceous stone ornaments (Bellina and Silapanth, 2006; Dussubieux and Bellina, 2017). At Khao Sek, as at Khao Sam Kaeo, the glass production involves similar Indian techniques for siliceous stones.

Wound beads are fairly rare. A few spherical opaque green beads were manufactured using this technique. Other beads were manufactured using technique that could not be identified.

Some beads were certainly imported as in the case of the orange beads with an annular shape that are present by the hundreds and red disc-shaped beads although found in much smaller number. Both types of beads are abundant at South and Southeast Asian sites although the red disc-shaped beads seem more common in South India and Sri Lanka (Dussubieux, 2001, 103) and the orange annular beads in Thailand (e.g. Higham and Kijngam, 2012; White, 1982) and Vietnam (Dussubieux, 2001, 103). The place of production for these beads is still unknown.

At least, one gold-foiled bead was recovered at Khao Sek and possibly a second one. Both specimens are collar beads. One of the beads is tabular and the other one is cylindrical. They can only have two origins: the Mediterranean area or Pakistan where a number of locally-manufactured gold-foiled beads were found (Dussubieux and Gratuze, 2003).

Another type of glass artifacts present at the site is bracelets. They come in a variety of shapes and colors. They are mostly transparent green $(\sim 80 \%)$, black $(\sim 14 \%)$ and turquoise blue $(\sim 5 \%)$. Other colors are orange, opaque green, opaque red and transparent dark blue but they are extremely rare and only a specimen or two is available in each of those colors. A large proportion of the bracelets has a semi-circular section (A or B type as defined at Khao Sam Kaeo, see Fig. 3) and are transparent green. Other sections include triangular, house-shaped, D or $\mathrm{C}$ sections. Different artifacts are distorted pieces of bracelets (Fig. 2). They would indicate that glass bracelets were manufactured at Khao Sek.

\subsection{Comparison with Khao Sam Kaeo}

Khao Sam Kaeo is a unique site in Southeast Asia as it yielded evidence of ornament production dating as early as the 4th c. BCE. This discovery changed the long and well-established view that glass ornaments in Southeast Asia at that time were exclusively imported from South Asia (Dussubieux and Bellina, 2017). The presence of a second glass working site $80 \mathrm{~km}$ away from Khao Sam Kaeo raises a range of new questions related to the chronology of the two sites, their roles and relationships.

Both sites have in common the presence of an important quantity of glass wastes and more especially of knapped glass fragments. Transparent green glass objects dominated the Khao Sam Kaeo glass 

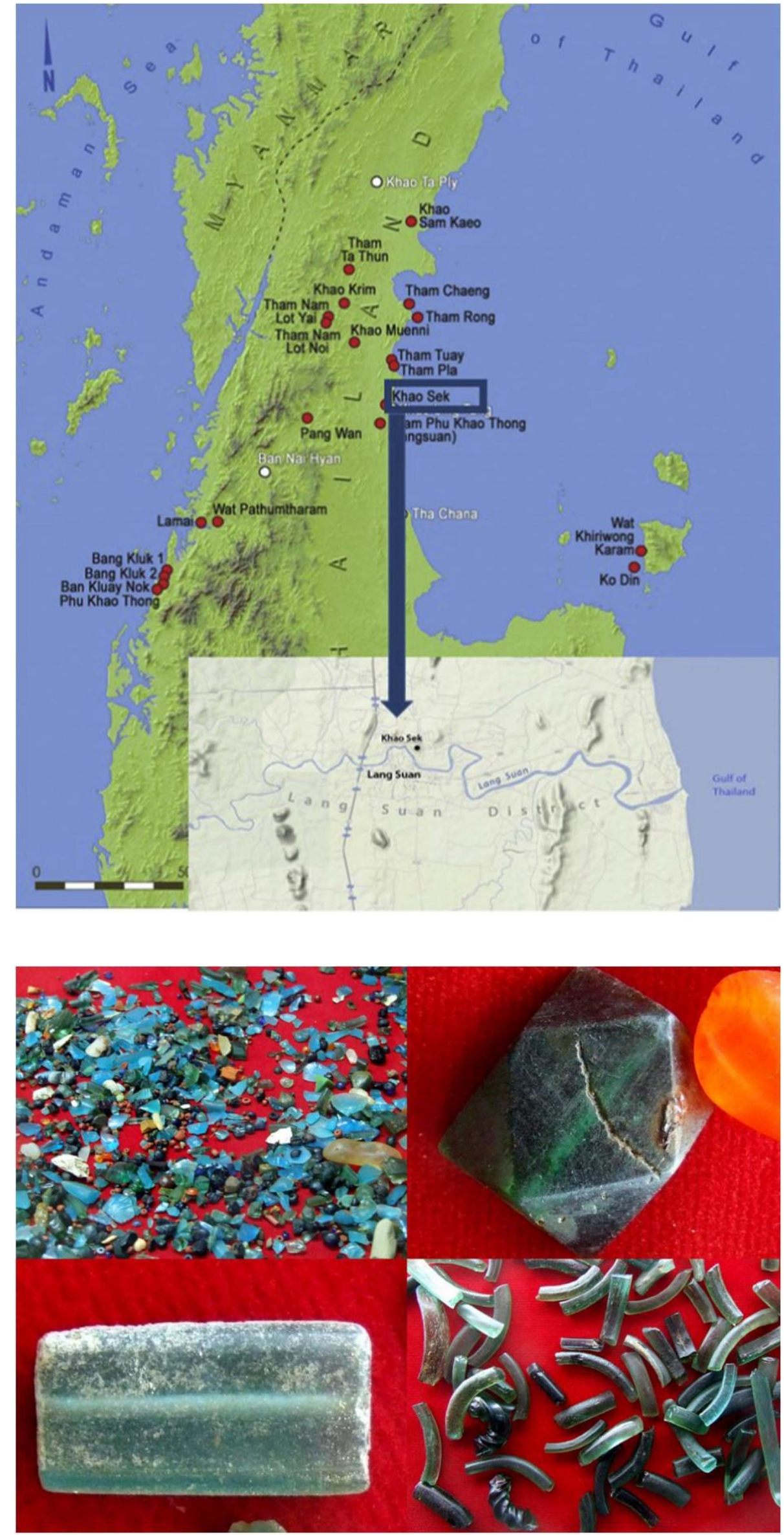

Fig. 1. Map indicating the location of Khao Sek.

Fig. 2. Glass material found at Khao Sek. Please, note that it was impossible to insert a scale in the pictures and they all have very different magnifications. Starting with the top left picture and going clockwise: beads and knapped glass waste, broken lapidary bead, bracelet fragments and hot worked waste, tabular lapidary bead. 


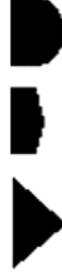 \\ : \\ Semi-circular A and B \\ House-shaped A and B \\ Triangular \\ D \\ Semi-circular C and D}

Fig. 3. Different shapes of bracelet section found at Khao Sam Kaeo and Khao Sek.

assemblage. It represented $87 \%$ of the bracelet, $87 \%$ of the knapped wastes and $90 \%$ of the hot worked wastes. All lapidary beads found at Khao Sam Kaeo were transparent green but very few were found there (16 in the excavations) compared to Kao Sek (> 100). Lapidary rectangular tabular beads are totally absent at Khao Sam Kaeo but fairly common at Khao Sek. At Khao Sek, more turquoise blue (47.5\%) and less transparent green (44\%) glass fragments were found among the waste. Color distributions for the bracelets are similar at both sites with approximately $80 \%$ of transparent green glass and approximately $5 \%$ of turquoise blue glass at Khao Sek; however, shape distributions for the bracelets are different for the two sites: at Khao Sam Kaeo, house shaped bracelets are the most common, followed by the semi-circular one. Other shapes were identified with 6 to 16 specimens in each category. At Khao Sek, even in the absence of an exact count, it was apparent, that the bracelets with a semi-circular section (B type) were the most common and the presence of distorted fragments of glass bracelets with a semi-circular and house shaped section indicate that both types of bracelets were made at the site. At both sites there is an important presence of drawn beads but no convincing evidence of their manufacture could be found.

It seems, according to the observations on the material from Khao Sam Kaeo and Khao Sek, that glass workers were using very similar technologies for the production of glass bracelets and possibly the production of lapidary glass beads. However, we noticed, very subtle differences in the productions from the two sites. As an illustration, at Khao Sam Kaeo there are many more house-shaped glass bracelets but no tabular beads.

\section{Compositions}

\subsection{Analytical technique}

The analyses were carried out at the Field Museum of Natural History in Chicago, USA, with a Analytik Jena Inductively Coupled Plasma - Mass Spectrometer (ICP-MS) connected to a New Wave UP213 laser for direct introduction of solid samples.

The parameters of the ICP-MS are optimized to ensure a stable signal with a maximum intensity over the full range of masses of the elements and to minimize oxides and double ionized species formation $\left(\mathrm{XO}^{+} / \mathrm{X}^{+}\right.$and $\mathrm{X}^{++} / \mathrm{X}^{+}<1$ to $\left.2 \%\right)$. For that purpose the argon flows, the RF power, the torch position, the lenses, the mirror and the detector voltages are adjusted using an auto-optimization procedure.

For better sensitivity, helium is used as a gas carrier in the laser. The choice of the parameters of the laser ablation not only will have an effect on the sensitivity of the method and the reproducibility of the measurements but also on the damage to the sample. To be able to determine elements with concentrations in the range of ppm and below while leaving a trace on the surface of the sample invisible to the naked eye, we use the single point analysis mode with a laser beam diameter of $80 \mu \mathrm{m}$, operating at $70 \%$ of the laser energy $(0.2 \mathrm{~mJ})$ and at a pulse frequency of $15 \mathrm{~Hz}$. A pre-ablation time of $20 \mathrm{~s}$ is set in order, first, to eliminate the transient part of the signal and, second, to be sure that a possible surface contamination or corrosion does not affect the results of the analysis. For each glass sample, the average of four measurements corrected from the blank is considered for the calculation of concentrations.

The isotope $\mathrm{Si} 29$ was used for internal standardization. Concentrations for major elements, including silica, are calculated assuming that the sum of their concentrations in weight percent in glass is equal to $100 \%$ (Gratuze, 1999). Two different series of external standards are used to measure major, minor and trace elements. The first series of external standards are standard reference materials (SRM) manufactured by NIST: SRM 610 and SRM 612. Both of these standards are soda-lime-silica glass doped with trace elements in the range of $500 \mathrm{ppm}$ (SRM 610) and $50 \mathrm{ppm}$ (SRM 612). Certified values are available for a very limited number of elements. Concentrations from Pearce et al. (1997) will be used for the other elements. The second series of standards were manufactured by Corning. Glass B and D are glasses that match compositions of ancient glass (Brill, 1999, vol. 2, p. 544).

The detection limits range from $10 \mathrm{ppb}$ to $1 \mathrm{ppm}$ for most of the elements. Accuracy ranges from 5 to $10 \%$ depending on the elements and their concentrations. A more detailed account of the performances of this technique can be found in Dussubieux et al. (2009).

\subsection{Samples}

Forty five samples were analyzed (Table 1).

\subsection{Results}

The 45 compositions obtained using LA-ICP-MS are available in Table 2. For a few samples with $\mathrm{SiO}_{2}>80 \%$, it is quite likely that some corroded material was analyzed but based on their whole composition, it was possible to assign them to a glass type.

The samples belong to three main glass groups:

- Potash glasses (22 specimens),

- M-Na-Al 3 glass (19 specimens),

- Soda plant ash glass (4 specimens).

\subsubsection{Potash glass}

A little more than half of the analyzed glass samples from Khao Sek are made of potash glass. In potash glasses, potash $\left(\mathrm{K}_{2} \mathrm{O}\right)$ is the most abundant constituent after silica and its concentration is around $15 \%$. Soda concentrations are generally low as are magnesia concentrations $(<1.5 \%)$. A relatively pure source of potash such as saltpetre was likely used. Different qualities of sand were added to the potash flux. Indeed, lime and alumina concentrations are very variable and based on compositions identified in South and Southeast Asia, three groups have been distinguished (Dussubieux and Gratuze, 2010; Lankton and Dussubieux, 2006, 2013):

$$
\begin{aligned}
& \text { - a high } \mathrm{Ca} \text { - low } \mathrm{Al}-\mathrm{K} \text { glass } \\
& \text { - a low } \mathrm{Ca} \text { - high } \mathrm{Al}-\mathrm{K} \text { glass } \\
& \text { - a moderate } \mathrm{Ca} \text { and } \mathrm{Al}-\mathrm{K} \text { glass. }
\end{aligned}
$$

Most of the high $\mathrm{Ca}-$ low $\mathrm{Al}-\mathrm{K}$ glass samples were identified at $\mathrm{Ban}$ Don Ta Phet, Thailand (4th - 3rd c. BCE). Potash glass found in India generally fits within the moderate $\mathrm{Al}$ and $\mathrm{Ca}-\mathrm{K}$ glass compositional range. From a general point of view, potash glasses from Southeast Asia have either moderate alumina and lime composition or low $\mathrm{Ca}$ - high Al composition. The origin of the potash glasses is still uncertain at this point even if a Chinese ( $\mathrm{Li}, 1999)$ or a Southeast Asian origin (Dussubieux and Pryce, 2016; Lankton et al., 2008) have been hypothesized. Potash glass is associated with sites that are usually dated no later than the 5 th $\mathrm{c}$. CE.

At Khao Sek, the compositions of the potash glass samples vary, with alumina and lime ranging respectively from 0.5 to $7 \%$ and 0.2 to 
Table 1

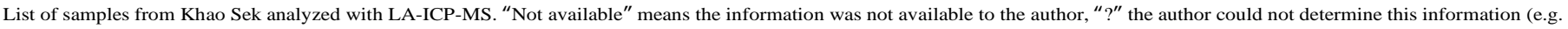
due to broken artifact), "n/a" this info is not relevant to the type of artifact.

\begin{tabular}{|c|c|c|c|c|c|}
\hline Reference & Artifact & Technology & Diaphanity & Color & Shape \\
\hline KSEK001 & Not available & Not available & Translucent & Dark blue & Not available \\
\hline KSEK002 & Not available & Not available & Translucent & Dark blue & Not available \\
\hline KSEK004 & Not available & Not available & Opaque & Red & Not available \\
\hline KSEK005 & Not available & Not available & Opaque & Yellow & Not available \\
\hline KSEK006 & Not available & Not available & Translucent & Dark blue & Not available \\
\hline KSEK007 & Not available & Not available & Translucent & Turquoise blue & Not available \\
\hline KSEK008 & Not available & Not available & Translucent & Turquoise blue & Not available \\
\hline KSEK009 & Not available & Not available & Transparent & green & Not available \\
\hline KSEK010 & Not available & Not available & Opaque & Red & Not available \\
\hline KSEK011 & Not available & Not available & Opaque & Red & Not available \\
\hline KSEK012 & Not available & Not available & Translucent & Dark blue & Not available \\
\hline KSEK013 & Not available & Not available & Opaque & Red & Not available \\
\hline KSEK015 & Not available & Not available & Transparent & Green & Not available \\
\hline KSEK016 & Not available & Not available & Transparent & Green & Not available \\
\hline KSEK018 & Waste & Knapped & Translucent & Turquoise blue & $\mathrm{n} / \mathrm{a}$ \\
\hline KSEK019 & Bead & $\mathrm{n} / \mathrm{a}$ & Translucent & Turquoise blue & $\mathrm{n} / \mathrm{a}$ \\
\hline KSEK020 & Bead & Drawn & Translucent & Turquoise blue & $\mathrm{n} / \mathrm{a}$ \\
\hline KSEK021 & Bead & Drawn & Translucent & Dark purple & Oblate \\
\hline KSEK022 & Bead & Drawn & Translucent & Dark purple & Oblate \\
\hline KSEK023 & Bead & Drawn & Translucent & Dark purple & Oblate \\
\hline KSEK024 & Bead & Drawn & Translucent & Dark purple & Oblate \\
\hline KSEK025 & Bead & Wound & Opaque & Green & Oblate \\
\hline KSEK026 & Bead & Drawn & Translucent & Turquoise blue & Oblate \\
\hline KSEK027 & Bead & Drawn & Translucent & Dark blue & Oblate \\
\hline KSEK028 & Bead & Drawn & Opaque & Red & Oblate \\
\hline KSEK029 & Bead & Drawn & Opaque & Red & Oblate \\
\hline KSEK030 & Bead & Drawn & Opaque & Green & Oblate \\
\hline KSEK031 & Bead & Drawn & Translucent & Turquoise blue & Oblate \\
\hline KSEK032 & Bead & Drawn & Translucent & Turquoise blue & Oblate \\
\hline KSEK033 & Bracelet (fragment) & $?$ & Opaque & Red & Not available \\
\hline KSEK034 & Bracelet (fragment) & $?$ & Opaque & Red & Not available \\
\hline 15KSek04 & Bead & Lapidary & Translucent & Green & Tabular rectangular \\
\hline 15Ksek06 & Waste & Knapped? & Translucent & Green with blue & $\mathrm{n} / \mathrm{a}$ \\
\hline 15 Ksek07 & Waste & Knapped? & Translucent & Green & $\mathrm{n} / \mathrm{a}$ \\
\hline 15 Ksek08 & Waste & Hot worked & Translucent & Green & $\mathrm{n} / \mathrm{a}$ \\
\hline 15Ksek09 & Pendant & $?$ & Translucent & Green & Double headed \\
\hline 15 Ksek 10 & Waste & Knapped? & Opaque & Red & $\mathrm{n} / \mathrm{a}$ \\
\hline 15KSek11 & Bead & Lapidary & Transparent & Colorless & Tabular rectangular \\
\hline $15 \mathrm{Ksek} 12$ & Bracelet & $?$ & Translucent & Green & Semi-circular B \\
\hline $15 \mathrm{Ksek} 16$ & Bracelet & $?$ & Opaque & Black & Semi-circular B \\
\hline 15KSek17 & Bead & Lapidary? & Translucent & Amber & Broken \\
\hline $15 \mathrm{KSek} 18$ & Bead & $?$ & Opaque & Green & Broken \\
\hline 15KSek19 & Bead & Drawn & Opaque & Orange & Square section, broken \\
\hline $15 \mathrm{KSek} 20$ & Waste & Knapped? & Translucent & Green & $\mathrm{n} / \mathrm{a}$ \\
\hline $15 \mathrm{KSek} 21$ & Waste & Knapped? & Opaque & Black & $\mathrm{n} / \mathrm{a}$ \\
\hline
\end{tabular}

7.2\%. Fig. 4 shows that samples fall in different groups: 4 samples with

$\mathrm{Al}_{2} \mathrm{O}_{3}$ concentrations higher than $3 \%$ and $\mathrm{CaO}$ concentrations lower than $2 \%$ are part of the m-K-Al glass group. Eight samples have moderate alumina $(<4 \%)$ and lime $(<4 \%)$ concentrations and are part of the m-K-Ca-Al group. A significant number of samples have compositions that do not match any of the groups listed above. Four samples have very low alumina and lime concentrations (respectively $\sim 0.5 \%$ and $1-1.3 \%)$. Other samples have relatively high lime concentrations $(>4 \%$ ) but do not fit very well in the $\mathrm{m}-\mathrm{K}-\mathrm{Ca}$ glass group due to alumina concentrations that are much higher than the usual for this glass type. At this point, it is uncertain whether those potash glass samples form sub-groups of their own or whether they are variations of one of the three sub-groups described above.

The four samples with very low alumina and lime are very similar small dark blue drawn beads. All the other groups contain samples that vary in nature and color. In the $\mathrm{m}-\mathrm{K}-\mathrm{Al}$ group, beads and a fragment of bracelet are either translucent/transparent turquoise and dark blue, transparent green and opaque yellow. The $\mathrm{m}-\mathrm{K}-\mathrm{Ca}-\mathrm{Al}$ group contains translucent or transparent turquoise and dark blue beads or wastes. The samples with higher lime and aluminum are translucent green and amber or opaque red. They are drawn or lapidary beads, pendant and waste.
We identified three different types of coloring recipes for the potash dark blue glass samples. The four beads (KSEK21 to 24) with low lime and alumina are colored by a combination of manganese and copper ( $\sim 2 \%$ for both constituent as oxides). Those beads contain $>5000$ ppm of barium which is much higher than in all the other potash dark blue beads. Two other samples (KSEK01 and 27) contain no significant amount of copper but $\sim 1 \%$ of $\mathrm{MnO}$. Their barium levels are $\sim 1000$ and $1400 \mathrm{ppm}$. These values are also much higher than in the glass without any significant amounts of manganese. This suggests that manganese and barium were added together to the glass. Sample KSEK01 belongs to the $\mathrm{m}-\mathrm{K}-\mathrm{Al}$ group and $\mathrm{KSEK} 027$ to the $\mathrm{m}-\mathrm{K}-\mathrm{Ca}-\mathrm{Al}$ group suggesting some connections between the craftsmen producing the two different potash glasses. Two beads (KSEK02 and 06) in the m-K-Ca-Al group are colored by cobalt $(\mathrm{Co}>1000 \mathrm{ppm})$. They contain very little manganese $(0.04 \%)$ but significant concentrations of copper $(0.8-1.4 \%)$. Arsenic is significantly more elevated in these two samples compared to the other potash dark blue ones.

Turquoise blue samples contain between 1.2 and $2.6 \%$ of $\mathrm{CuO}$. Other glass samples colored with copper are the red glass samples (KSEK13 and 28). Their $\mathrm{CuO}$ concentrations are slightly higher ( 3\%). They also have higher $\mathrm{MgO}, \mathrm{P}_{2} \mathrm{O}_{5}$ and $\mathrm{CaO}$ concentrations compared to the other potash glasses. Higher concentrations of these constituents 
Table 2

Composition of the glass samples from Khao Sek in \%w of oxide and ppm of element. $<\mathrm{dl}$ stands for below the detection limit.

\begin{tabular}{|c|c|c|c|c|c|c|c|c|c|c|c|c|c|c|c|c|c|c|c|c|c|}
\hline & KSEK001 & $\begin{array}{l}\text { KSE- } \\
\text { K002 }\end{array}$ & $\begin{array}{l}\text { KSE- } \\
\text { K004 }\end{array}$ & $\begin{array}{l}\text { KSE- } \\
\text { K005 }\end{array}$ & KSEK006 & $\begin{array}{l}\text { KSE- } \\
\text { K007 }\end{array}$ & KSEK008 & $\begin{array}{l}\text { KSE- } \\
\text { K009 }\end{array}$ & KSEK010 & $\begin{array}{l}\text { KSE- } \\
\text { K011 }\end{array}$ & $\begin{array}{l}\text { KSE- } \\
\text { K013 }\end{array}$ & $\begin{array}{l}\text { KSE- } \\
\text { K015 }\end{array}$ & $\begin{array}{l}\text { KSE- } \\
\text { K016 }\end{array}$ & $\begin{array}{l}\text { KSE- } \\
\text { K018 }\end{array}$ & $\begin{array}{l}\text { KSE- } \\
\text { K019 }\end{array}$ & KSEK020 & $\begin{array}{l}\text { KSE- } \\
\text { K021 }\end{array}$ & $\begin{array}{l}\text { KSE- } \\
\text { K022 }\end{array}$ & $\begin{array}{l}\text { KSE- } \\
\text { K023 }\end{array}$ & KSEK024 & $\begin{array}{l}\text { KSE- } \\
\text { K025 }\end{array}$ \\
\hline & Potash & Potash & $\begin{array}{l}\text { m-Na-Al } \\
3\end{array}$ & Potash & Potash & Potash & Potash & $\begin{array}{l}\text { m-Na-Al } \\
3\end{array}$ & $\begin{array}{l}\mathrm{m}-\mathrm{Na}-\mathrm{Al} \\
3\end{array}$ & $\begin{array}{l}\text { m-Na-Al } \\
3\end{array}$ & Potash & $\begin{array}{l}\text { m-Na-Al } \\
3\end{array}$ & $\begin{array}{l}\text { m-Na-Al } \\
3\end{array}$ & Potash & Potash & Potash & Potash & Potash & Potash & Potash & $\mathrm{v}-\mathrm{Na}-\mathrm{Ca}$ \\
\hline $\mathrm{SiO} 2$ & $79.7 \%$ & $82.6 \%$ & $65.1 \%$ & $49.5 \%$ & $73.9 \%$ & $85.2 \%$ & $77.4 \%$ & $76.0 \%$ & $68.8 \%$ & $68.4 \%$ & $63.6 \%$ & $67.0 \%$ & $66.7 \%$ & $82.6 \%$ & $75.2 \%$ & $75.9 \%$ & $78.0 \%$ & $79.4 \%$ & $78.7 \%$ & $76.4 \%$ & $63.4 \%$ \\
\hline $\mathrm{Na} 2 \mathrm{O}$ & $0.57 \%$ & $0.17 \%$ & $12.8 \%$ & $0.34 \%$ & $0.12 \%$ & $1.32 \%$ & $1.44 \%$ & $6.91 \%$ & $8.42 \%$ & $8.49 \%$ & $0.5 \%$ & $15.6 \%$ & $15.3 \%$ & $0.51 \%$ & $1.05 \%$ & $1.39 \%$ & $0.64 \%$ & $0.59 \%$ & $0.61 \%$ & $0.72 \%$ & $13.9 \%$ \\
\hline $\mathrm{MgO}$ & $0.22 \%$ & $0.25 \%$ & $1.62 \%$ & $0.13 \%$ & $0.54 \%$ & $0.50 \%$ & $0.38 \%$ & $1.31 \%$ & $1.83 \%$ & $1.86 \%$ & $2.8 \%$ & $1.2 \%$ & $1.1 \%$ & $0.33 \%$ & $0.55 \%$ & $0.44 \%$ & $0.15 \%$ & $0.14 \%$ & $0.14 \%$ & $0.18 \%$ & $3.51 \%$ \\
\hline $\mathrm{Al} 2 \mathrm{O} 3$ & $3.10 \%$ & $2.52 \%$ & $6.72 \%$ & $3.09 \%$ & $1.91 \%$ & $1.31 \%$ & $1.12 \%$ & $8.03 \%$ & $7.94 \%$ & $8.11 \%$ & $1.9 \%$ & $7.9 \%$ & $7.5 \%$ & $1.79 \%$ & $1.77 \%$ & $3.94 \%$ & $0.55 \%$ & $0.53 \%$ & $0.54 \%$ & $0.51 \%$ & $3.69 \%$ \\
\hline P2O5 & $0.13 \%$ & $0.13 \%$ & $1.02 \%$ & $0.09 \%$ & $0.33 \%$ & $0.24 \%$ & $0.20 \%$ & $0.51 \%$ & $1.37 \%$ & $1.27 \%$ & $1.1 \%$ & $0.4 \%$ & $0.3 \%$ & $0.29 \%$ & $0.47 \%$ & $0.28 \%$ & $0.16 \%$ & $0.16 \%$ & $0.14 \%$ & $0.15 \%$ & $0.22 \%$ \\
\hline K2O & $13.9 \%$ & $10.8 \%$ & $4.67 \%$ & $9.30 \%$ & $17.8 \%$ & $6.66 \%$ & $14.8 \%$ & $3.69 \%$ & $4.24 \%$ & $4.23 \%$ & $18.8 \%$ & $2.8 \%$ & $4.4 \%$ & $10.1 \%$ & $15.3 \%$ & $13.7 \%$ & $12.5 \%$ & $11.5 \%$ & $11.8 \%$ & $13.7 \%$ & $4.29 \%$ \\
\hline $\mathrm{CaO}$ & $0.33 \%$ & $1.33 \%$ & $4.27 \%$ & $0.22 \%$ & $3.85 \%$ & $2.41 \%$ & $2.53 \%$ & $2.08 \%$ & $3.52 \%$ & $3.52 \%$ & $6.16 \%$ & $2.63 \%$ & $2.13 \%$ & $2.57 \%$ & $3.21 \%$ & $2.25 \%$ & $1.16 \%$ & $1.04 \%$ & $1.05 \%$ & $1.39 \%$ & $6.20 \%$ \\
\hline $\mathrm{MnO}$ & $0.96 \%$ & $0.04 \%$ & $0.15 \%$ & $0.02 \%$ & $0.04 \%$ & $0.02 \%$ & $0.03 \%$ & $0.09 \%$ & $0.17 \%$ & $0.17 \%$ & $0.1 \%$ & $0.1 \%$ & $0.1 \%$ & $0.02 \%$ & $0.03 \%$ & $0.04 \%$ & $1.85 \%$ & $1.78 \%$ & $1.82 \%$ & $1.78 \%$ & $0.07 \%$ \\
\hline $\mathrm{Fe} 2 \mathrm{O} 3$ & $0.45 \%$ & $0.52 \%$ & $0.99 \%$ & $0.43 \%$ & $0.46 \%$ & $0.23 \%$ & $0.28 \%$ & $1.04 \%$ & $1.47 \%$ & $1.51 \%$ & $1.0 \%$ & $2.1 \%$ & $2.1 \%$ & $0.46 \%$ & $0.43 \%$ & $0.62 \%$ & $0.19 \%$ & $0.23 \%$ & $0.26 \%$ & $0.28 \%$ & $0.33 \%$ \\
\hline $\mathrm{CuO}$ & $0.10 \%$ & $1.44 \%$ & $2.20 \%$ & $0.10 \%$ & $0.84 \%$ & $1.25 \%$ & $1.27 \%$ & $0.03 \%$ & $1.95 \%$ & $2.09 \%$ & $3.6 \%$ & $0.0 \%$ & $0.0 \%$ & $1.29 \%$ & $1.80 \%$ & $1.23 \%$ & $2.16 \%$ & $2.05 \%$ & $2.10 \%$ & $2.09 \%$ & $0.46 \%$ \\
\hline $\mathrm{SnO} 2$ & $0.04 \%$ & $0.01 \%$ & $0.009 \%$ & $3.48 \%$ & $0.01 \%$ & $0.007 \%$ & $0.02 \%$ & $0.02 \%$ & $0.02 \%$ & $0.01 \%$ & $0.02 \%$ & $0.002 \%$ & $0.005 \%$ & $0.002 \%$ & $0.01 \%$ & $0.003 \%$ & $0.03 \%$ & $0.03 \%$ & $0.03 \%$ & $0.03 \%$ & $0.60 \%$ \\
\hline $\mathrm{PbO}$ & $0.27 \%$ & $0.004 \%$ & $0.009 \%$ & $33.3 \%$ & $0.002 \%$ & $0.02 \%$ & $0.003 \%$ & $0.006 \%$ & $0.006 \%$ & $0.007 \%$ & $0.005 \%$ & $0.005 \%$ & $0.004 \%$ & $0.008 \%$ & $0.004 \%$ & $0.006 \%$ & $0.02 \%$ & $0.02 \%$ & $0.02 \%$ & $0.02 \%$ & $3.02 \%$ \\
\hline $\mathrm{Li}$ & 14 & 7.1 & 16 & 3.1 & 12 & 3.1 & 10 & 20 & 30 & 29 & 11 & 23 & 21 & 2.7 & 6.9 & 7.1 & 3.3 & 2.3 & 2.5 & 3.2 & 17 \\
\hline $\mathrm{Be}$ & 1.9 & 2.3 & 2.4 & 1.2 & 1.2 & 1.6 & 1.3 & 3.0 & 2.2 & 2.1 & 1.6 & 2.9 & 2.1 & 0.4 & 0.4 & 0.9 & 0.7 & 1.0 & 0.9 & 0.9 & 0.6 \\
\hline B & 83 & 67 & 107 & 30 & 83 & 82 & 71 & 115 & 184 & 112 & 263 & 139 & 124 & 92 & 89 & 89 & 78 & 74 & 75 & 69 & 142 \\
\hline $\mathrm{Sc}$ & 4.1 & 1.2 & 5.8 & 1.9 & 1.0 & 0.8 & 0.9 & 5.3 & 6.8 & 7.0 & 3.7 & 8.0 & 7.3 & 3.6 & 3.3 & 4.8 & 1.5 & 1.6 & 1.9 & 2.1 & 2.9 \\
\hline $\mathrm{Ti}$ & 757 & 435 & 1906 & 660 & 547 & 384 & 332 & 2089 & 2260 & 2323 & 775 & 1963 & 1883 & 436 & 565 & 859 & 138 & 125 & 129 & 134 & 914 \\
\hline $\mathrm{v}$ & 42 & 13 & 60 & 16 & 12 & 12 & 12 & 69 & 56 & 56 & 19 & 64 & 60 & 11 & 9.2 & 18 & 13 & 12 & 13 & 13 & 13 \\
\hline $\mathrm{Cr}$ & 17 & 14 & 39 & 23 & 11 & 10 & 11 & 45 & 53 & 54 & 19 & 47 & 44 & 18 & 15 & 19 & 4.5 & 6.4 & 5.5 & 7.5 & 10 \\
\hline $\mathrm{Ni}$ & 29 & 290 & 38 & 15 & 264 & 40 & 28 & 18 & 51 & 48 & 66 & 19 & 20 & 21 & 58 & 33 & 21 & 21 & 21 & 21 & 9.4 \\
\hline Co & 171 & 1232 & 24 & 4.0 & 1108 & 58 & 28 & 6.5 & 16 & 16 & 81 & 7.8 & 6.2 & 13 & 54 & 17 & 104 & 99 & 102 & 99 & 3.6 \\
\hline $\mathrm{Zn}$ & 34 & 27 & 112 & 27 & 27 & 29 & 22 & 83 & 136 & 134 & 118 & 90 & 78 & 19 & 28 & 29 & 37 & 39 & 37 & 36 & 19 \\
\hline As & 24 & 453 & 21 & 3.4 & 747 & 52 & 25 & 2.3 & 14 & 10 & 13 & $<\mathrm{dl}$ & $<\mathrm{dl}$ & 32 & 148 & 52 & 102 & 86 & 98 & 85 & 1.7 \\
\hline $\mathrm{Rb}$ & 430 & 111 & 87 & 170 & 182 & 116 & 153 & 142 & 128 & 123 & 87 & 94 & 256 & 86 & 147 & 124 & 93 & 86 & 93 & 97 & 89 \\
\hline $\mathrm{Sr}$ & 38 & 67 & 172 & 14 & 84 & 102 & 103 & 157 & 183 & 187 & 200 & 122 & 111 & 74 & 63 & 87 & 38 & 36 & 37 & 47 & 302 \\
\hline $\mathrm{Zr}$ & 130 & 69 & 204 & 112 & 94 & 88 & 74 & 222 & 222 & 236 & 38 & 198 & 181 & 71 & 84 & 103 & 35 & 19 & 16 & 17 & 153 \\
\hline $\mathrm{Nb}$ & 4.4 & 2.3 & 9.5 & 5.1 & 2.7 & 2.0 & 1.7 & 11 & 12 & 12 & 3.0 & 10 & 10 & 1.6 & 2.5 & 3.9 & 1.0 & 0.8 & 0.8 & 0.9 & 4.4 \\
\hline $\mathrm{Ag}$ & 0.9 & 5.0 & 2.5 & 40 & 0.5 & 0.7 & 1.2 & 1.6 & 2.9 & 1.4 & 5.0 & 1.0 & 0.6 & 2.2 & 1.5 & 1.3 & 9.0 & 10 & 10 & 8.1 & 3.3 \\
\hline $\mathrm{Sb}$ & 1.4 & 4.6 & 4.7 & 2.0 & 9.0 & 2.8 & 2.1 & 0.9 & 3.5 & 1.5 & 3.3 & 0.8 & 2.2 & 0.7 & 2.7 & 0.8 & 8.2 & 7.6 & 7.5 & 7.3 & 27 \\
\hline $\mathrm{Cs}$ & 2.7 & 2.0 & 3.5 & 2.1 & 1.3 & 0.5 & 1.0 & 4.6 & 6.4 & 5.9 & 1.6 & 4.3 & 4.8 & 0.5 & 0.8 & 1.2 & 0.4 & 0.4 & 0.3 & 0.6 & 1.4 \\
\hline $\mathrm{Ba}$ & 994 & 122 & 371 & 51 & 216 & 84 & 105 & 398 & 469 & 486 & 250 & 333 & 314 & 96 & 191 & 239 & 5217 & 5180 & 5542 & 5548 & 654 \\
\hline $\mathrm{La}$ & 13 & 11 & 32 & 10 & 13 & 13 & 11 & 38 & 35 & 36 & 7.0 & 30 & 27 & 8.4 & 10 & 15 & 3.7 & 3.5 & 3.8 & 3.7 & 13 \\
\hline $\mathrm{Ce}$ & 62 & 23 & 63 & 21 & 24 & 24 & 22 & 75 & 69 & 71 & 14 & 64 & 57 & 16 & 20 & 28 & 17 & 16 & 18 & 18 & 24 \\
\hline Pr & 3.6 & 2.7 & 7.5 & 2.3 & 2.8 & 2.9 & 2.4 & 8.9 & 8.4 & 8.5 & 1.7 & 7.2 & 6.5 & 1.9 & 2.4 & 3.4 & 0.9 & 0.8 & 0.9 & 1.0 & 3.2 \\
\hline $\mathrm{Ta}$ & 0.8 & 1.5 & 0.8 & 0.4 & 0.2 & 0.2 & 0.2 & 1.1 & 1.2 & 1.2 & 0.4 & 1.0 & 0.8 & 0.1 & 0.2 & 0.4 & 0.2 & 0.3 & 0.2 & 0.2 & 0.5 \\
\hline Y & 8.6 & 8.3 & 23 & 8.4 & 8.6 & 7.6 & 7.9 & 25 & 24 & 25 & 5.7 & 24 & 21 & 6.6 & 7.4 & 11 & 3.1 & 3.2 & 3.1 & 3.0 & 8.3 \\
\hline $\mathrm{Bi}$ & 0.4 & 2.8 & 3.5 & 0.7 & 1.7 & 3.9 & 1.6 & 0.9 & 1.5 & 1.5 & 4.0 & 0.4 & 0.4 & 4.1 & 1.6 & 2.5 & 11 & 10 & 10 & 10 & 2.7 \\
\hline U & 1.1 & 1.0 & 68 & 2.6 & 1.1 & 1.0 & 1.2 & 163 & 29 & 26 & 2.5 & 61 & 55 & 0.6 & 0.8 & 4.7 & 0.5 & 0.5 & 0.5 & 0.5 & 1.4 \\
\hline W & 0.7 & 2.6 & 2.0 & 0.4 & 1.2 & 1.2 & 0.6 & 3.5 & 3.0 & 3.2 & 2.6 & 2.2 & 1.9 & 1.7 & 0.8 & 1.5 & 0.8 & 0.7 & 0.8 & 0.7 & 0.3 \\
\hline Mo & 20 & 101 & 12 & 1.1 & 36 & 27 & 23 & 8.2 & 14 & 14 & 13 & 10 & 8.1 & 56 & 25 & 43 & 27 & 24 & 28 & 25 & 0.9 \\
\hline $\mathrm{Nd}$ & 12 & 9.1 & 26 & 7.8 & 10 & 9.3 & 8.8 & 31 & 29 & 30 & 5.9 & 26 & 23 & 6.7 & 8.7 & 12 & 3.2 & 3.0 & 3.2 & 3.3 & 11 \\
\hline $\mathrm{Sm}$ & 2.7 & 1.8 & 5.4 & 1.6 & 2.0 & 2.0 & 1.8 & 6.3 & 6.1 & 6.1 & 1.3 & 5.4 & 4.4 & 1.5 & 1.8 & 2.5 & 0.9 & 1.1 & 0.8 & 1.0 & 2.5 \\
\hline $\mathrm{Eu}$ & 0.7 & 0.3 & 0.9 & 0.3 & 0.4 & 0.3 & 0.3 & 1.2 & 1.3 & 1.1 & 0.4 & 0.9 & 0.9 & 0.2 & 0.3 & 0.5 & 0.3 & 0.3 & 0.3 & 0.3 & 0.6 \\
\hline $\mathrm{Gd}$ & 2.2 & 1.7 & 4.6 & 1.5 & 1.8 & 1.5 & 1.5 & 5.5 & 5.0 & 5.4 & 1.1 & 4.5 & 3.9 & 1.2 & 1.5 & 2.1 & 0.8 & 0.8 & 0.8 & 0.9 & 2.0 \\
\hline $\mathrm{Tb}$ & 0.4 & 0.2 & 0.7 & 0.3 & 0.3 & 0.3 & 0.3 & 0.9 & 0.8 & 0.8 & 0.4 & 0.7 & 0.6 & 0.2 & 0.2 & 0.3 & 0.1 & 0.1 & 0.1 & 0.1 & 0.3 \\
\hline Dy & 2.1 & 1.6 & 4.2 & 1.4 & 1.6 & 1.5 & 1.5 & 4.8 & 4.8 & 5.0 & 1.8 & 4.3 & 3.5 & 1.1 & 1.5 & 2.2 & 0.7 & 0.9 & 0.7 & 0.8 & 2.0 \\
\hline Но & 0.5 & 0.3 & 0.9 & 0.4 & 0.3 & 0.3 & 0.3 & 1.0 & 1.0 & 1.0 & 0.5 & 0.8 & 0.7 & 0.2 & 0.3 & 0.5 & 0.2 & 0.2 & 0.2 & 0.2 & 0.4 \\
\hline $\mathrm{Er}$ & 1.1 & 1.0 & 2.3 & 1.0 & 0.9 & 0.9 & 0.8 & 3.0 & 2.8 & 2.7 & 0.9 & 2.6 & 2.1 & 0.6 & 0.8 & 1.2 & 0.4 & 0.4 & 0.4 & 0.4 & 1.2 \\
\hline $\mathrm{Tm}$ & 0.3 & 0.4 & 0.3 & 0.2 & 0.2 & 0.1 & 0.1 & 0.4 & 0.4 & 0.4 & 0.1 & 0.4 & 0.4 & 0.1 & 0.1 & 0.2 & 0.1 & 0.1 & 0.1 & 0.1 & 0.2 \\
\hline $\mathrm{Yb}$ & 1.3 & 1.0 & 2.5 & 1.2 & 0.9 & 0.8 & 1.0 & 2.9 & 2.6 & 3.1 & 1.0 & 2.4 & 2.3 & 0.6 & 0.8 & 1.6 & 0.5 & 0.7 & 0.5 & 0.5 & 1.3 \\
\hline
\end{tabular}


Table 2 (continued)

\begin{tabular}{|c|c|c|c|c|c|c|c|c|c|c|c|c|c|c|c|c|c|c|c|c|c|}
\hline & KSEK001 & $\begin{array}{l}\text { KSE- } \\
\text { K002 }\end{array}$ & $\begin{array}{l}\text { KSE- } \\
\text { K004 }\end{array}$ & $\begin{array}{l}\text { KSE- } \\
\text { K005 }\end{array}$ & KSEK006 & $\begin{array}{l}\text { KSE- } \\
\text { K007 }\end{array}$ & KSEK008 & $\begin{array}{l}\text { KSE- } \\
\text { K009 }\end{array}$ & KSEK010 & $\begin{array}{l}\text { KSE- } \\
\text { K011 }\end{array}$ & $\begin{array}{l}\text { KSE- } \\
\text { K013 }\end{array}$ & $\begin{array}{l}\text { KSE- } \\
\text { K015 }\end{array}$ & $\begin{array}{l}\text { KSE- } \\
\text { K016 }\end{array}$ & $\begin{array}{l}\text { KSE- } \\
\text { K018 }\end{array}$ & $\begin{array}{l}\text { KSE- } \\
\text { K019 }\end{array}$ & KSEK020 & $\begin{array}{l}\text { KSE- } \\
\text { K021 }\end{array}$ & $\begin{array}{l}\text { KSE- } \\
\text { K022 }\end{array}$ & $\begin{array}{l}\text { KSE- } \\
\text { K023 }\end{array}$ & KSEK024 & $\begin{array}{l}\text { KSE- } \\
\text { K025 }\end{array}$ \\
\hline Lu & 0.2 & 0.2 & 0.3 & 0.1 & 0.1 & 0.1 & 0.1 & 0.6 & 0.5 & 0.4 & 0.3 & 0.4 & 0.3 & 0.1 & 0.1 & 0.2 & 0.1 & 0.1 & 0.1 & 0.1 & 0.2 \\
\hline $\mathrm{Hf}$ & 3.8 & 2.4 & 6.4 & 3.1 & 2.8 & 2.9 & 2.6 & 7.5 & 7.4 & 7.3 & 1.3 & 6.0 & 5.3 & 1.8 & 2.5 & 3.3 & 1.2 & 1.0 & 0.7 & 0.8 & 6.6 \\
\hline Th & 4.0 & 5.0 & 13 & 4.4 & 5.8 & 4.3 & 3.7 & 16 & 16 & 16 & 3.2 & 14 & 12 & 2.7 & 4.5 & 6.6 & 1.5 & 1.5 & 1.6 & 2.0 & 6.6 \\
\hline
\end{tabular}

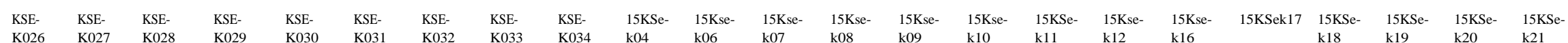

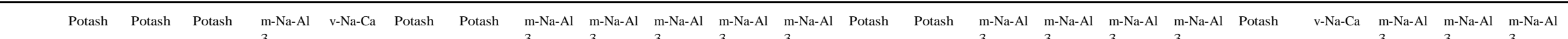

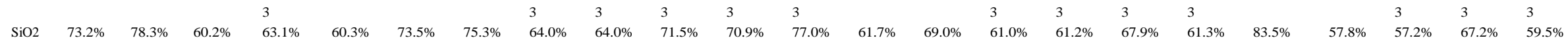

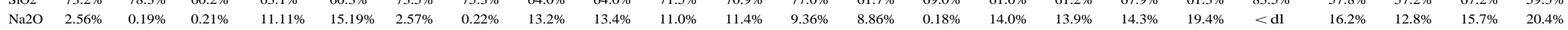

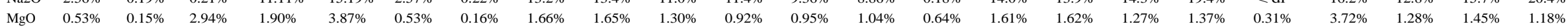

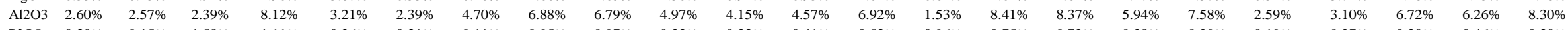

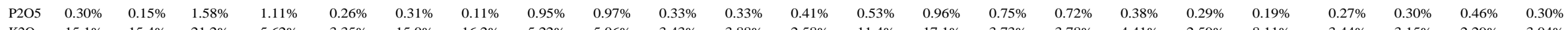

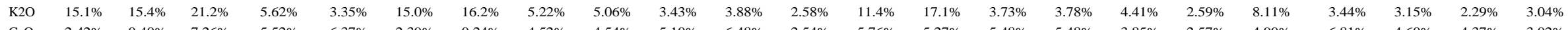

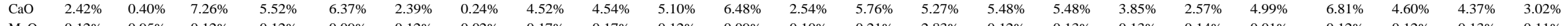

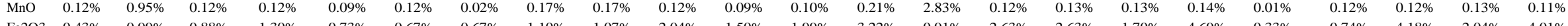

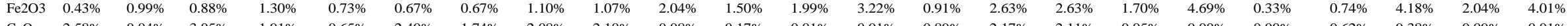

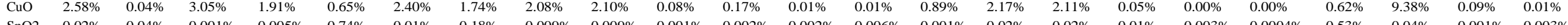

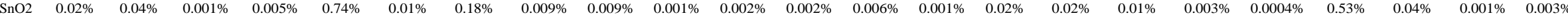

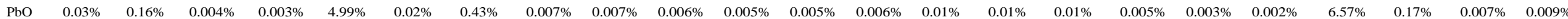

\begin{tabular}{|c|c|c|c|c|c|c|c|c|c|c|c|c|c|c|c|c|c|c|c|c|c|c|c|}
\hline $\mathrm{Li}$ & 15 & 22 & 9.5 & 23 & 18 & 12 & 8.4 & 16 & 15 & 21 & 17 & 13 & 205 & 8.2 & 21 & 21 & 16 & 17 & 5.2 & 19 & 17 & 16 & 20 \\
\hline $\mathrm{Be}$ & 145 & 0.6 & 0.6 & 1.6 & 0.5 & 142 & 0.6 & 1.3 & 1.4 & 2.0 & 1.4 & 1.3 & 5.2 & 0.7 & 1.5 & 1.5 & 1.2 & 1.5 & 0.5 & 0.3 & 1.4 & 1.2 & 1.8 \\
\hline B & 81 & 110 & 68 & 147 & 152 & 84 & 68 & 101 & 105 & 77 & 112 & 144 & 27 & 63 & 122 & 124 & 86 & 98 & 59 & 153 & 70 & 141 & 100 \\
\hline $\mathrm{Sc}$ & 3.2 & 6.2 & 5.8 & 9.6 & 5.2 & 4.3 & 6.5 & 8.6 & 8.7 & 6.8 & 4.7 & 6.7 & 9.5 & 3.5 & 8.3 & 8.2 & 5.8 & 7.0 & 3.3 & 2.7 & 6.2 & 6.4 & 8.1 \\
\hline $\mathrm{Ti}$ & 736 & 626 & 1384 & 2631 & 981 & 678 & 1012 & 2267 & 2245 & 1656 & 986 & 1533 & 2220 & 524 & 2004 & 2020 & 1447 & 1875 & 657 & 763 & 1657 & 1559 & 2102 \\
\hline $\mathrm{V}$ & 19 & 30 & 34 & 62 & 15 & 18 & 19 & 61 & 60 & 64 & 43 & 77 & 52 & 26 & 72 & 72 & 48 & 74 & 12 & 14 & 44 & 69 & 82 \\
\hline $\mathrm{Cr}$ & 13 & 23 & 30 & 54 & 22 & 21 & 25 & 46 & 52 & 41 & 21 & 37 & 3.8 & 11 & 44 & 39 & 28 & 44 & 5.6 & 9.3 & 32 & 33 & 49 \\
\hline $\mathrm{Ni}$ & 83 & 38 & 33 & 34 & 13 & 82 & 15 & 38 & 36 & 20 & 62 & 17 & 19 & 27 & 46 & 46 & 13 & 12 & 3.1 & 25 & 256 & 14 & 15 \\
\hline Co & 86 & 206 & 11 & 11 & 7.3 & 81 & 3.2 & 24 & 23 & 21 & 202 & 20 & 10 & 190 & 12 & 12 & 27 & 4.9 & 1.3 & 19 & 119 & 5.5 & 5.8 \\
\hline $\mathrm{Zn}$ & 34 & 30 & 153 & 110 & 153 & 32 & 35 & 120 & 116 & 63 & 71 & 74 & 1939 & 88 & 100 & 101 & 57 & 86 & 14 & 251 & 53 & 65 & 83 \\
\hline As & 153 & 3.5 & 24 & 8.0 & 5.5 & 142 & 353 & 12 & 11 & 6.6 & 207 & 17 & $<\mathrm{dl}$ & 0.2 & 13 & 10 & 13 & $<\mathrm{dl}$ & $<\mathrm{dl}$ & 31 & 516 & $<\mathrm{dl}$ & $<\mathrm{dl}$ \\
\hline $\mathrm{Rb}$ & 128 & 343 & 88 & 95 & 51 & 110 & 364 & 78 & 76 & 93 & 82 & 125 & 237 & 100 & 96 & 95 & 79 & 81 & 97 & 48 & 83 & 70 & 94 \\
\hline $\mathrm{Sr}$ & 92 & 28 & 282 & 209 & 274 & 79 & 8.4 & 155 & 155 & 180 & 199 & 125 & 365 & 115 & 149 & 149 & 119 & 110 & 72 & 279 & 113 & 133 & 124 \\
\hline $\mathrm{Zr}$ & 96 & 103 & 54 & 227 & 169 & 80 & 143 & 191 & 175 & 241 & 137 & 199 & 280 & 62 & 153 & 145 & 166 & 186 & 89 & 172 & 207 & 179 & 211 \\
\hline $\mathrm{Nb}$ & 3.3 & 3.4 & 4.4 & 11 & 4.1 & 2.7 & 5.5 & 8.7 & 8.4 & 10 & 5.8 & 9.4 & 21 & 2.7 & 10 & 11 & 7.7 & 10 & 3.6 & 3.8 & 10 & 8.4 & 11 \\
\hline $\mathrm{Ag}$ & 5.3 & 0.3 & 6.4 & 3.1 & 8.7 & 4.6 & 11 & 2.5 & 2.4 & 0.2 & 0.1 & 0.3 & 0.1 & 1.0 & 3.1 & 3.0 & 0.1 & 0.1 & 0.1 & 7.8 & 8.3 & 0.1 & 0.1 \\
\hline $\mathrm{Sb}$ & 8.9 & 0.3 & 3.8 & 4.6 & 177 & 7.3 & 22 & 4.5 & 3.6 & 0.4 & 3.0 & 1.1 & 0.5 & 0.4 & 4.8 & 4.9 & 3.5 & 0.7 & 0.5 & 43 & 26 & 1.7 & 1.0 \\
\hline $\mathrm{Cs}$ & 4.1 & 1.2 & 1.4 & 4.1 & 0.9 & 3.3 & 3.3 & 2.8 & 2.8 & 3.5 & 2.7 & 3.5 & 2.1 & 0.5 & 4.4 & 4.4 & 3.0 & 3.9 & 1.1 & 0.8 & 3.7 & 2.8 & 4.6 \\
\hline $\mathrm{Ba}$ & 238 & 1404 & 332 & 446 & 350 & 183 & 48 & 367 & 358 & 430 & 424 & 357 & 780 & 3968 & 402 & 395 & 315 & 370 & 97 & 307 & 328 & 322 & 428 \\
\hline $\mathrm{La}$ & 15 & 12 & 9.1 & 33 & 8.2 & 11 & 9.5 & 26 & 26 & 36 & 22 & 33 & 54 & 9.0 & 30 & 30 & 26 & 30 & 13 & 8.2 & 32 & 30 & 35 \\
\hline $\mathrm{Ce}$ & 31 & 53 & 19 & 69 & 16 & 24 & 22 & 53 & 51 & 68 & 43 & 67 & 106 & 29 & 60 & 60 & 51 & 58 & 26 & 15 & 62 & 63 & 69 \\
\hline $\operatorname{Pr}$ & 3.5 & 3.0 & 2.1 & 7.9 & 1.8 & 2.5 & 2.1 & 6.3 & 6.0 & 8.6 & 5.4 & 8.0 & 13 & 2.4 & 7.3 & 7.4 & 6.4 & 7.3 & 3.2 & 1.9 & 7.9 & 7.5 & 8.7 \\
\hline Тa & 0.4 & 0.3 & 0.6 & 0.9 & 0.3 & 0.2 & 0.4 & 0.7 & 0.7 & 1.0 & 0.5 & 0.9 & 2.5 & 0.2 & 0.9 & 0.9 & 0.8 & 1.1 & 0.3 & 0.3 & 0.9 & 0.8 & 1.0 \\
\hline Y & 10 & 10 & 6.9 & 25 & 5.9 & 9 & 10 & 20 & 20 & 26 & 15 & 23 & 36 & 6.9 & 24 & 23 & 20 & 22 & 11 & 6.3 & 21 & 22 & 25 \\
\hline $\mathrm{Bi}$ & 4.0 & 0.2 & 467 & 0.8 & 22 & 2.7 & 58 & 4.6 & 2.9 & 0.4 & 0.9 & 0.3 & 0.6 & 1.3 & 3.2 & 3.3 & 0.2 & 0.1 & 0.1 & 14 & 8.3 & 0.2 & 0.1 \\
\hline U & 7.6 & 0.9 & 0.7 & 37 & 2.4 & 5.0 & 1.3 & 54 & 50 & 59 & 131 & 204 & 9.2 & 0.8 & 111 & 115 & 50 & 185 & 1.4 & 1.4 & 36 & 139 & 206 \\
\hline W & 1.2 & 0.2 & 1.5 & 2.1 & 0.4 & 0.9 & 0.4 & 1.7 & 1.7 & 1.7 & 2.0 & 2.6 & 1.1 & 0.6 & 2.3 & 1.9 & 1.9 & 2.1 & 2.6 & 0.6 & 1.7 & 1.8 & 2.3 \\
\hline Mo & 25 & 1.3 & 17 & 10 & 1.0 & 28 & 0.5 & 11 & 11 & 10 & 27 & 21 & 0.3 & 14 & 18 & 13 & 12 & 5.0 & 95 & 1.2 & 9.4 & 16 & 5.7 \\
\hline $\mathrm{Nd}$ & 12 & 11 & 8.0 & 28 & 6.6 & 9.1 & 7.6 & 22 & 22 & 29 & 18 & 27 & 43 & 9.0 & 24 & 24 & 20 & 24 & 10 & 6.2 & 26 & 24 & 28 \\
\hline $\mathrm{Sm}$ & 2.6 & 2.6 & 1.7 & 5.7 & 1.4 & 1.9 & 1.6 & 4.5 & 4.4 & 5.9 & 3.9 & 5.5 & 8.3 & 1.9 & 4.8 & 4.8 & 4.0 & 4.6 & 2.0 & 1.3 & 5.0 & 4.9 & 5.7 \\
\hline $\mathrm{Eu}$ & 0.4 & 0.6 & 0.4 & 1.0 & 0.3 & 0.3 & 0.3 & 0.8 & 0.7 & 0.9 & 0.6 & 0.9 & 1.3 & 0.5 & 0.8 & 0.8 & 0.7 & 0.8 & 0.4 & 0.3 & 0.8 & 0.8 & 1.0 \\
\hline $\mathrm{Gd}$ & 2.3 & 2.1 & 1.6 & 4.9 & 1.3 & 1.6 & 1.4 & 3.7 & 3.5 & 5.1 & 3.0 & 4.6 & 6.8 & 1.9 & 4.1 & 4.1 & 3.5 & 4.0 & 1.9 & 1.2 & 4.1 & 4.0 & 4.7 \\
\hline
\end{tabular}



as the m-Na-Al glasses (Dussubieux et al., 2011, 2010). It was suggested that an ingredient containing calcium, magnesium, phosphorus and maybe other elements were added in order to facilitate the reduction of the copper and to facilitate the red color.

Three other glass samples are either translucent green or amber. One of the green samples (15KSEK09) contains copper $(0.9 \%)$ and manganese (2.8\%). The other green sample (15KSEK08) contains 3.2\% of $\mathrm{Fe}_{2} \mathrm{O}_{3}$. For the amber sample (15KSEK17) no specific element seems to be present in higher quantities.

\subsection{2. $\mathrm{m}-\mathrm{Na}-\mathrm{Al} 3$ glass}

In South and Southeast Asia, one of the most abundant glasses is called mineral soda-alumina or $\mathrm{m}-\mathrm{Na}-\mathrm{Al}$ glass and results from the mix of a soda-rich efflorescence called reh and a high alumina sand (Brill, 1987). Alumina that has concentrations varying from 5 to $15 \%$ in this glass is introduced by poorly refined sand. The sand composition is similar to that of granite and contains a small amount of potash and lime, and relatively high concentrations of iron, titanium and other trace elements, such as rare earth elements (Dussubieux, 2001; Dussubieux and Gratuze, 2004). Trace elements allow for the separation of the m-Na-Al glass type into sub-groups (Dussubieux et al., 2010). To determine what type of m-Na-Al glass was found in Khao Sek, Principal Component Analysis (PCA) was conducted using $\mathrm{MgO}, \mathrm{Zr}, \mathrm{Sr}$, $\mathrm{Ba}, \mathrm{U}$ and $\mathrm{Cs}$ concentrations measured in $\mathrm{m}-\mathrm{Na}-\mathrm{Al} 1$ glass samples (southern India/Sri Lanka), m-Na-Al 2 glass samples (western India) and in m-Na-Al 3 glass samples (northern India) (Fig. 5). The 19 samples from Khao Sek with a m-Na-Al composition are more similar to glass m-Na-Al 3. This glass was identified at Kopia, in the region of Uttar Pradesh (Kanungo, 2006; Kanungo and Misra, 2004; Kanungo and Shinde, 2005). The finding of hundreds of glass chunks and ceramic fragments coated with glass suggested that glass was manufactured at this site; however, most of the glass manufacturing evidence was found in disturbed deposits that could not be dated. Charcoal found in a furnace pit yielded a date of $120 \mathrm{cal}$ CE $( \pm 120)$ (Kanungo et al., 2010). Kanungo (2010:473) suggests that "glass might have been produced here (in Kopia) from the NBP period onwards". The NBP period or the Northern Black Polished Ware culture corresponds to 700-200 BCE.

The m-Na-Al 3 glass samples found at Khao Sek include knapped wastes, lapidary beads and bracelets fragments. Four colors were identified: orange, opaque red, black and transparent green. These colors were obtained using two main constituents (Fig. 6): iron and copper. In samples with very little copper, iron $\left(\mathrm{Fe}_{2} \mathrm{O}_{3}\right)$ ranges from either 1 to $2 \%$ in the transparent green glass or higher than $4 \%$ in the black glass samples. In both cases, the quantities of copper are very low. In the red glass samples, copper is $\sim 2 \%$ with variable iron concentrations $(1-2.6 \%)$. The orange glass sample has more copper $(9.4 \%)$ and more iron $(4.2 \%)$.

\subsubsection{Soda plant ash}

The third type of glass (also called v-Na-Ca glass) found at Khao Sek is manufactured using soda plant ashes. This type of glass presents many similarities with glass made in the Middle-East from the Sasanian period through the Islamic period. Samples with a v-Na-Ca composition are separated in two groups. Some of them are either vessel fragments with a clear Middle-Eastern influence regarding their typology or glass beads. Both types of artifacts appear at later sites dated mostly around the 7 th to the 10th c. CE. Direct importation from the Middle-East seems quite likely for that type of material. Earlier material, only available in the forms of beads, was found at Sri Lankan sites (dated from the 4th c. BCE to the 2nd c. CE) but also in Southeast Asia at sites such as Angkor Borei (200 BCE-200AD) or Oc Eo (50-250 CE and 400-550 CE). Because of the early dating of this material, a MiddleEastern provenance is more questionable and the source of this glass material remains uncertain at this point (Dussubieux, 2001; Lankton 


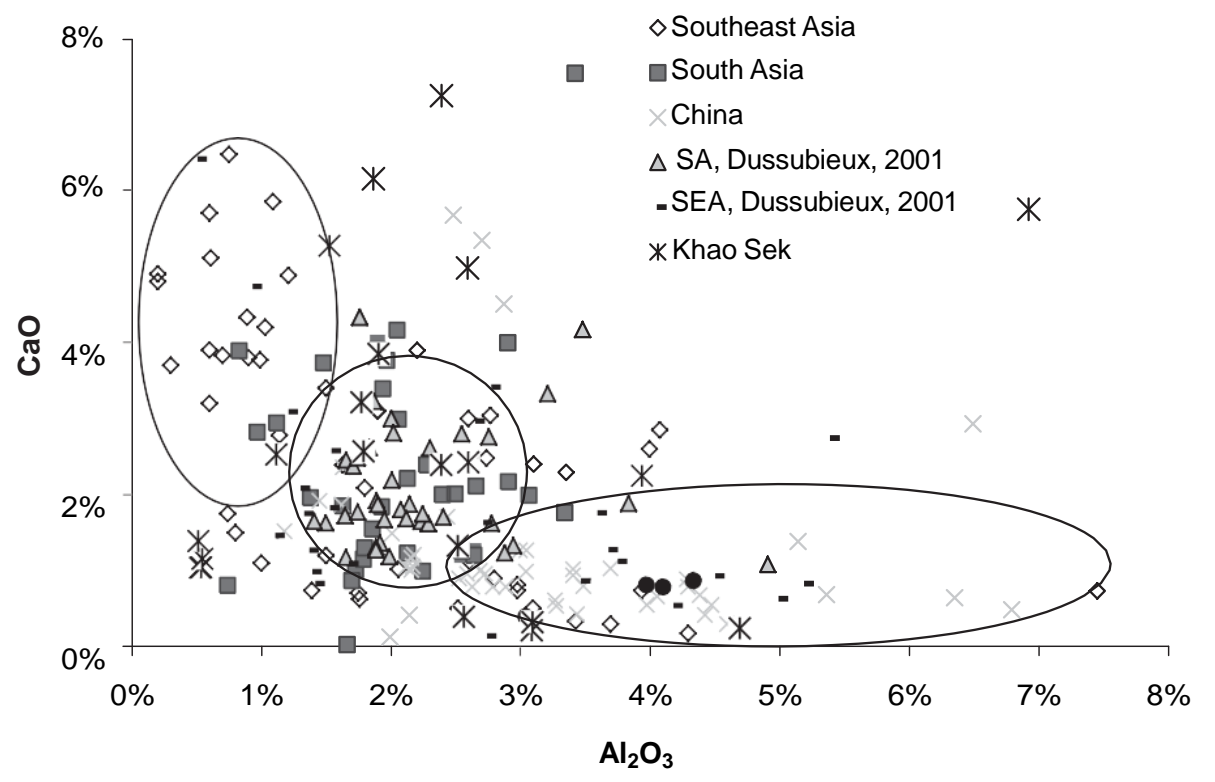

Fig. 4. Alumina and lime concentrations in potash glass artifacts found in South Asia (Brill, 1987, 1999; Glover and Henderson, 1995; Francis, 1998; Lal, 1952; Lamb, 1965; Singh, 1989; Subramanian, 1950; Varshney, 1950, Varshneya et al., 1988, Dussubieux, 2001), Southeast Asia (Brill, 1999; Basa, 1991; Salisbury and Glover, 1997, Dussubieux, 2001), China (Qishan, 1996; Li, 1999; Fukang, 1991; Zhang et al., 2005, 2004) and Khao Sek. The ellipses are for an easy visualisation of the three main potash glass groups.

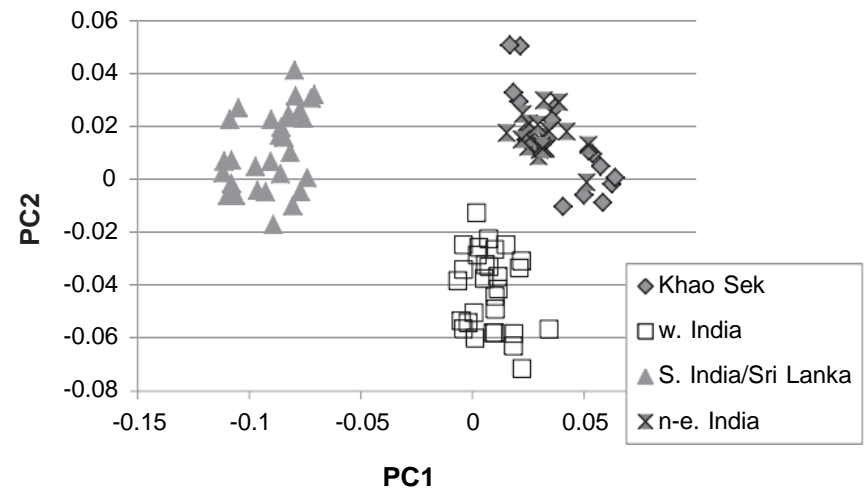

Fig. 5. Principal components 1 and 2 obtained with PCA including $\mathrm{MgO}, \mathrm{Sr}, \mathrm{Ba}, \mathrm{U}$ and $\mathrm{Cs}$ concentrations measured in glass samples found in Southern India/Sri Lanka (Dussubieux, unpublished), in Western India (Dussubieux et al., 2008) and North-eastern India (Dussubieux and Kanungo, 2013). PC 1 and 2 account for $83 \%$ of the variability of the dataset.

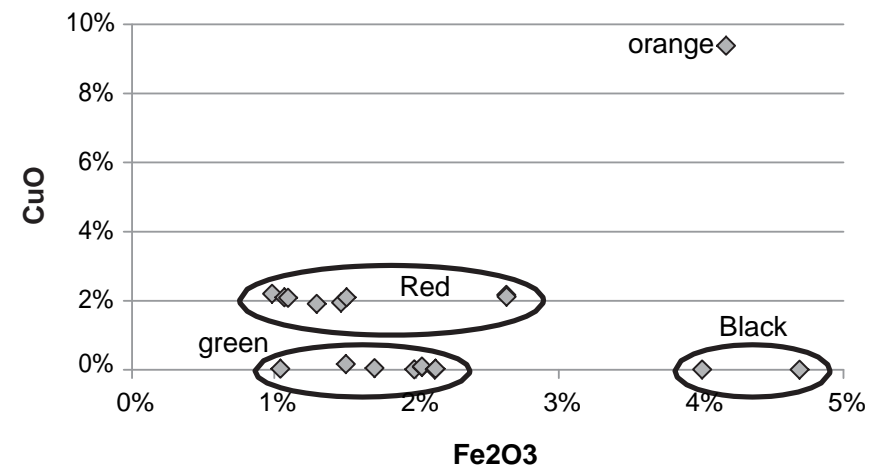

Fig. 6. Bi-plot showing the $\mathrm{Fe}_{2} \mathrm{O}_{3}$ and $\mathrm{CuO}$ concentrations in the Khao Sek m-Na-Al 3 glass samples.

and Dussubieux, 2006).

Samples made from soda plant ash are either dark blue (KSEK12) or opaque green (KSEK25 and 30, 15KSKEK18). The dark blue sample contains significant quantities of cobalt (300 ppm), copper $(0.4 \%)$ and arsenic $(300 \mathrm{ppm})$. The opaque green glass beads have higher concentrations of tin $\left(0.5-0.7 \%\right.$ of $\left.\mathrm{SnO}_{2}\right)$ and lead (3.0 to $6.6 \%$ of $\left.\mathrm{PbO}\right)$ that
Table 3

Proportions of the different glass types present at Khao Sam Kaeo and Khao Sek.

\begin{tabular}{lll}
\hline & Khao Sam Kaeo & Khao Sek \\
\hline Potash & $50 \%$ & $49 \%$ \\
m-Na-Al 3 & $28 \%$ & $42 \%$ \\
v-Na-Ca & $0 \%$ & $9 \%$ \\
Other & $22 \%$ & $0 \%$ \\
\hline
\end{tabular}

might have been used as lead stannate that is a yellow opacifier. The presence of copper $(0.5-0.7 \%$ of $\mathrm{CuO})$ and lead stannate in the glass are certainly causing the green opaque aspect of the glass.

\section{Glass type distribution in Southeast Asia}

\subsection{Comparison with Khao Sam Kaeo}

Typological similarities were noted earlier between the type of material found at Khao Sam Kaeo and at Khao Sek. It is therefore essential to compare the compositions found at both sites (Table 3). It is first important to note that a larger quantity of glass was analyzed at Khao Sam Kaeo (162 specimens) compared to Khao Sek (45). At Khao Sam Kaeo, $50 \%$ of the analyzed glass samples had a potash composition. The m-Na-Al 3 glass samples were then the second most abundant with $28 \%$. At Khao Sek, the proportion of the $\mathrm{m}-\mathrm{Na}-\mathrm{Al} 3$ and potash glass are fairly similar (respectively 42 and $49 \%$ ). Other glass types identified at Khao Sam Kaeo include m-Na-Al 1, m-Na-Ca-Al, mixed-alkali for those with the highest proportions (Dussubieux and Bellina, 2017).

Among the m-Na-Al 3 glass found at Khao Sam Kaeo, only three colors were represented: translucent green (the most preeminent), opaque red and more rarely black. At Khao Sek, those three colors are present but opaque orange is also available. The range of colors in the potash glass group at Khao Sam Kaeo is wider, including colorless and orange glasses that are absent at Khao Sek. However, the number of potash samples is much lower at Khao Sek and a larger sample might have revealed more colors in the Khao Sek potash glass. At Khao Sek, 3 lapidary beads were analyzed and two of them have a m-Na-Al 3 composition and one is manufactured from potash glass. The four analyzed lapidary beads from Khao Sam Kaeo were all manufactured with m-Na-Al 3 glass.

At Khao Sek, the presence of dark blue potash glass containing 
cobalt and arsenic is worth noticing as this combination is rare and was only identified at the site of Khao Sam Kaeo and Ban Don Ta Phet (4th c. BCE). It seems that this cobalt was used early on (4th -2 nd c. BCE) and then replaced by another type of cobalt (Co-Mn-Ba) that is usually present in most of the dark blue glass with a potash composition found in South and Southeast Asia (Dussubieux, 2016).

Overall the two groups of glass samples have a lot in common with identical major glass types in addition of similar ornament typology. This suggests connection between the two places.

\subsection{Comparison with other South and Southeast Asian sites}

At Khao Sam Kaeo, as at Khao Sek, the dominant glass types are potash and m-Na-Al 3. This is usually associated with the very early glass network that operated around the 4th to the 2nd c. BCE (Dussubieux and Gratuze, 2010) and that includes also Ban Don Ta Phet (Thailand) and Giong Ca Vo (Vietnam). More recently, research undertaken on glass beads found in the Samon Valley in Myanmar, showed that this network extended into this region although beads types and color palette were very different there compared to what was found in Thailand and in Vietnam, suggesting the existence of two exchange networks: a larger network encompassing Northeastern India, modern Myanmar and Thailand used to trade raw glass and a series of more regional networks used to trade finished glass products (Dussubieux and Pryce, 2016).

Among the different sites (Ban Don Ta Phet and Giong Ca Vo) and areas (Samon Valley) mentioned above, Khao Sek and Khao Sam Kaeo are the only sites presenting evidence of glass bead and bracelet manufacturing, setting these two sites apart.

The most distinctive ornaments found at Khao Sek and Khao Sam Kaeo are the lapidary beads and bracelets. Lapidary beads have been identified in South and Southeast Asia. The sites of Ahichchhatra and Kausambi in North India yielded translucent green beads with a prismatic shape whereas hexagonal bi-conical beads were more common in South India (Basa, 1992). A possible production site could be Arikamedu (Basa, 1992); however no solid evidence for a glass lapidary bead-production centre in South Asia has been found so far. Chakraborty (1995-96) mentions the similarity between some hexagonal green beads found at Harinarayanpur, Chandraketugarh, and Deulpota (West Bengal) and at Arikamedu. In Southeast Asia, lapidary beads were found in Thailand at Ban Chiang (Labbé, 1985), and Peter Francis (2001) mentions the use of lapidary techniques at Ban Don Luang. Ban Don Ta Phet yielded a variety of beads that were certainly manufactured using the lapidary techniques (Glover, 1990). LA-ICP-MS analysis showed that they have a potash composition (Dussubieux, unpublished analysis).

As far as bracelets are concerned, their presence is attested at Ban Don Ta Phet (Glover, 1990) and at a Mimotien site in Cambodia (Haidle, 2001). Similar material was recovered during excavation at certain Sa Huynh-related sites in Vietnam, sites such as Giong Ca Vo, Phu Hoa, and Go Ma Voi (Van Thang and Hien, 1997:32; Fontaine, 1972:437-8; Nguyen Kim Dung et al., 1995; Reinecke et al., 2002:77, Fig. 59, $\mathrm{n}^{\circ}$ 9). Possibly similar specimens were recovered in the Oc Eo area in Vietnam (Malleret, 1962:257, $\mathrm{n}^{\circ} 1189$ ) and from Chamber B in Manunggul Cave, Tabon Caves, Palawan Island, Philippines (Fox, 1970:118).

\section{Discussion}

As expressed earlier, the presence of two very early glass workshops displaying similar industrial models at Khao Sam Khaeo and Khao Sek located $80 \mathrm{~km}$ apart and whose productions are comparable raises a range of questions related to their respective role and relationships. At Khao Sam Kaeo, it was established that possible glass workers from Northeastern India settled there to manufacture glass ornaments. The difference in the object styles and colors found at both sites is so subtle that it clearly indicates that the people that worked at both locations were the members of a same group of artisans, trained within the same tradition. However the presence of two glass workshops producing ornaments in a same region whereas workshops in Southeast Asia are so rare was at first puzzling. This glass industry standardization is also observable for other industries in both sites, strengthening the idea of close links between them and raising the question of how similar hybrid industrial models were implemented. The hypothesis of the circulation between polities of the same confederation of trading polities controlling different networks and trans-peninsular routes is suggested to account for this standardization (Bellina, in press a, 2017; Bellina, this issue $\mathrm{c}, \mathrm{d}$ ). As in the case of the hard stone industry, the standardized production between the two sites is hypothesized to result from the transfer of artisans in the context of a hierarchically and complementary-organized confederation of trade polities (see Bellina on stone industry organization and general discussion, Bellina, this issue a,b). Khao Sam Kaeo, larger but also whose settlement configuration is more complex (rampart, compounds, cosmopolitan population) and craft productions bigger, is interpreted as the leading polity acting as the central market at an «international » level where foreign merchants settled and craftsmen concentrated (Bellina, this issue a,b). The two contemporaneous workshops operated with artisans circulating between the two sites. What made this transfer possible? Either the original group split into two groups (or more) to start distinct glass workshops within this confederation, or they trained apprentices who moved to Khao Sek. Under which conditions this move took place can only be speculated. If one agrees to postulate that in the context of trading polities ornaments and their industry could have played the role of political currency, then the transfer of attached artisans could have taken the form of an honorific "gift" made by the central place polity's leader to weave his network of clients to secure the loyalty of the attached polity. This transfer could have been a means for the central place polity's leader to weave a network of clients as well as to secure his marketplace supply, thus ensuring the wealth of his polity and its charisma. Artisans, either those who initially settled at Khao Sam Kaeo or who were trained by them, could have moved to Khao Sek in the frame of these strategic political and economic bonds.

\section{Conclusion}

The analysis of the glass from Khao Sek and its comparison with Khao Sam Kaeo provides unprecedented evidence for a regional craft standardization involving hybrid industrial models. In both workshops, bracelets and lapidary beads were made using similar Indian techniques and glass. The high alumina glass type relates more specifically to the northeastern part of the Indian subcontinent. Meanwhile, the diffusion of both workshops manufactured artifacts were directed in the hinterland and in the South China Sea towards other communities involved in trade at different levels (Bellina, in press a). The confederation of trading polities controlled the local resources exploitation (tin in particular see Pryce et al., 2017; Pryce and Bellina, this issue) and transpeninsular routes (Bellina, this issue a), possibly through the exchange of glass and other locally-manufactured products. As suggested in the case of the hard stone industry, it is possible that the diffusion of these hybrid products but also of industrial models were transferred between associated trade polities. This could thus correspond to what Manguin called "Umland", a wider « hinterland » connected by its riverine and maritime network (2002). The transfer through this broader hinterland provides a tentative framework to explain the diffusion of similar panregional culture with the South China Sea Sphere of Interaction (Bellina, in press a).

To sum up, along other industries, the analysis and comparison of the glass industry at Khao Sek with Khao Sam Kaeo brings unprecedented data on the economic and political organization of the early trading polities of the South China Sea and a possible explanation for the diffusion of a pan-regional culture. 


\section{Conflict of interest}

We wish to confirm that there are no known conflicts of interest associated with this publication and there has been no significant financial support for this work that could have influenced its outcome.

\section{Acknowledgements}

This work was supported in part by a grant from the French Minister of Foreign Affair to Bérénice Bellina.

\section{References}

Basa, K.K., 1991. The Westerly Trade of Southeast Asia from c. 400 B.C. to c. 500 A.D with special reference to glass beads. Unpublished $\mathrm{PhD}$ thesis. The Institute of Archaeology, University College London, University of London.

Basa, K.K., 1992. Early glass beads in India. Southeast Asian Stud. 8, 91-104.

Bellina, B., 2007. Cultural Exchange Between India and Southeast Asia: Production an Distribution of Hard Stone Ornaments, VIc. BC-VIc. AD. Editions de la Maison des sciences de l'Homme, Paris.

Bellina, B., 2014. Maritime Silk Roads' ornament industries: socio-political practices and cultural transfers in the South China Sea. Camb. Archaeol. J. 24 (3), 345-377.

Bellina, B., 2017a. Southeast Asian evidence for early maritime exchange and trade-related polities. In: Higham, C.W.F., Kim, N. (Eds.), The Oxford Handbook of Southeast Asian Archaeology. Oxford University Press (in press a).

Bellina, B., 2017. Khao Sam Kaeo: a cosmopolitan port-city or city-state in the South China Sea. In: Bellina, B. (Ed.), Khao Sam Kaeo: A Late Prehistoric Early Port-city Between the Indian Ocean and the South China Sea. Ecole française d'Extrême-Orient, Paris, pp. 624-665

Bellina, B., 2017c. Development of maritime Trade Polities and Regional production systems between the Bay of Bengal and the South China Sea: the Khao Sek excavations and industries' studies contribution. Archaeol. Res. Asia (this issue a).

Bellina, B., 2017d. The elaboration of political models in maritime Southeast Asia and of pan-regional culture: contribution from Khao Sek stone ornament craft system study. Archaeol. Res. Asia (this issue b)

Bellina, B., Silapanth, Praon, 2006. Khao Sam Kaeo and the Upper Thai Peninsula: understanding the mechanism of early trans-asiatic trade and cultural exchange. In: Bacus, E.A., Glover, I.C., Pigott, V.C. (Eds.), Uncovering Southeast Asia's Past-Selected Papers From the Tenth Biennial Conference of the European Association of Southeast Asian Archaeologists, London, 14th-17th September 2004 National University Press, Singapore, pp. 379-392.

Blench, R., 2017. Restructuring our understanding of the South China Sea interaction sphere: the evidence from multiple disciplines. In: Calanca, P., Yi-ch'ang, Liu, Muyard, F. (Eds.), Taiwan Maritime Landscapes From Neolithic to Early Modern Times. Ecole française d'Extrême-Orient and the Institute of History and Philology, Academia Sinica, Taipei (in press)

Brill, R.H., 1987. Chemical analyses of some early Indian glasses. In: Bhardwaj, H.C. (Ed.), Archaeometry of Glass - Proceedings of the Archaeometry Session of the XIV International Congress on Glass - 1986 (Part I). Indian Ceramic Society, New Delhi, India, pp. 1-25.

Brill, R.H., 1999. Chemical Analyses of Early Glasses. vol 2 The Corning Museum of Glass, New York.

Carter, A.K., Dussubieux, L., 2016. Geologic provenience analysis of agate and carnelian beads using laser ablation-inductively coupled plasma-mass spectrometry (LA-ICPMS): a case study from Iron Age Cambodia and Thailand. J. Archaeol. Sci. Rep. 6, $321-331$

Chakraborty, S., 1995. Beads from Chandraketugarh. Pratna Samiksha 4 and 5, 32-53.

Dung, Nguyen Kim, Can, Trinh, Van Thang, Dan, Hien, Vu Quoc, Hau, Nguyen Thi, 1995. Ornaments from jar burial sites in Can Gio. Khao Co Hoc 2, 27-46 (in Vietnamese).

Dussubieux, L., 2001. L'Apport de l'ablation laser couplée à l'ICP-MS à l'étude du verre archéologique de l'Océan Indien. Unpublished PhD thesis. Université d'Orléans, France.

Dussubieux, L., 2016. Potash glass: a view from South and Southeast Asia. In: Gan, Fuxi, Henderson, Julian, Li, Quinghui (Eds.), Recent Progress of Scientific Research on Ancient Glass and Glaze. Series on Archaeology and History of Science in China (Book 2) World Scientific Publishing Company, pp. 95-111.

Dussubieux, L., Bellina, B., 2017. Glass from an early Southeast Asian producing and trading centre. In: Bellina (Ed.), Khao Sam Kaeo: A Late Prehistoric Early Port-city Between the Indian Ocean and the South China Sea. Ecole française d'Extrême-Orient, Paris, pp. 549-585.

Dussubieux, L., Gratuze, B., 2003. Nature et origine des objets en verre retrouvés à Begram (Afghanistan) et à Bara (Pakistan). In: Bopearachchi, O., Landes, C., Sachs, C. (Eds.), De l'Indus à l'Oxus: Archéologie de l'Asie Centrale, Lattes. Association Imago, Musée de Lattes, pp. 315-323.

Dussubieux, L., Gratuze, B., 2004. Non destructive characterization of glass beads: application to the study of glass trade between India and Southeast Asia. In: 9th International Conference of the European Association of Southeast Asian Archaeologists, Sigtuna, May 27th-June 2nd, pp. 135-148.

Dussubieux, L., Gratuze, B., 2010. Glass in Southeast Asia. In: Bellina, B., Bacus, E.A., Pryce, T.O., Wisseman, C.J. (Eds.), 50 years of Archaeology in Southeast Asia: Essays in Honour of Ian Glover. River Books, pp. 247-259.

Dussubieux, L., Kanungo, A.K., 2013. Trace Element Analysis of Glass from Kopia. In
Kanungo, A.K. (Ed.), Glass in Ancient India: Excavations at Kopia. KCHR, Triruvananthapuram, pp. 360-366.

Dussubieux, L., Pryce, O., 2016. Myanmar's role in Iron Age interaction networks linking Southeast Asia and India: recent glass and copper-base metal exchange research from the Mission Archéologique Française au Myanmar. J. Archaeol. Sci. Rep. 5, 598-614.

Dussubieux, L., Robertshaw, P., Glascock, M.D., 2009. LA-ICP-MS analysis of African glass beads: laboratory inter-comparison with an emphasis on the impact of corrosion on data interpretation. Int. J. Mass Spectrom. 284, 152-161.

Dussubieux, L., Gratuze, B., Blet-Lemarquand, M., 2010. Mineral soda alumina glass: occurrence and meaning. J. Archaeol. Sci. 37, 1645-1655.

Dussubieux, L., Blet-Lemarquand, M., Gratuze, B., 2011. Innovation dans les techniques de coloration: les verres rouges et orange en Asie du Sud. In: Actes du deuxième colloque international de l'association Verre \& Histoire. Association Verre \& Histoire, Paris. http://www.verre-histoire.org/colloques/innovations/pages/p101_01_ dussubieux.html (accessed 12/29/2016).

Dussubieux, L., Kusimba, C.M., Gogte, V., Kusimba, S.B., Gratuze, B., Oka, R., 2008. The trading of ancient glass beads: new analytical data from South Asian and East African soda-alumina glass beads. Archaeom. 50 (5), 797-821.

Fontaine, H., 1972. Nouveau champ de jarres dans la province de Long-Khanh. Bulletin de la société d'études indochinoises 47 (3), 398-446.

Fox, R.B., 1970. The Tabon Caves. In: Archaeological Explorations and Excavations on the Palawan Island. Monograph of the National Museum, No.1, Philippines. Manila.

Francis Jr., P., 1998. Analyses of Indo-Pacific beads. The Margaretologist 111 (25), 9-14.

Francis Jr., P., 2001. Asia's Maritime Bead Trade From ca. 300 BC to the Present. University of Hawai'i Press, Honolulu.

Fukang, Zhang, 1991. Scientific studies of early glasses excavated in China. In: Brill, R.H., Martin, J.H. (Eds.), Scientific Research in Early Chinese Glass, Proceedings of the Archaeometry of Glass Sessions of the 1984 International Symposium on Glass, Beijing, September 7, 1984 With Supplementary Papers. The Corning Museum of Glass, NY, pp. 157-165.

Glover, I., 1990. Ban Don Ta Phet: the 1984-85 excavation. In: Glover, I., Glover, E. (Eds.), Southeast Asia Archaeology 1986, Proceedings of the First Conference of the Association of Southeast Asian Archaeologist in Western Europe, Institute of Archaeology, University College, London, 8th-10th, September 1986. vol. 561. BAR International Series, Oxford, pp. 139-182.

Glover, I., Henderson, J., 1995. Early glass in South and South East Asia and China. In: Scott, R., Guy, J. (Eds.), South East Asia and China: Art, Interaction and Commerce. School of Oriental \& African Studies, London, pp. 141-170.

Gratuze, B., 1999. Obsidian characterization by laser ablation ICP-MS and its application to prehistoric trade in the Mediterranean and the Near East: sources and distribution of obsidian within the Aegean and Anatolia. J. Archaeol. Sci. 26, 869-881.

Gupta, S., 2005. The Bay of Bengal Interaction Sphere (1000 BC-AD 500). Bull. IndoPacific Prehist. Assoc. 25, 21-30.

Haidle, M.N., 2001. Fragments of glass bangles from Krek 52/62 and their implications for the dating of the Mimotien Culture. Asian Perspect. 40 (2), 195-208.

Higham, C.F.W., Kijngam, A. (Eds.), 2012. The Excavation of Ban Non Wat: The Iron Age, Summary and Conclusions, the Origins of the Civilization of Angkor, Volume VI. The Thai Fine Arts Department, Bangkok.

Hung, H.-C., Nguyen, K.D., Bellwood, P., Carson, M.T., 2013. Coastal connectivity: longterm trading networks across the South China Sea. J. Island Coast. Archaeol. 8, 384-404.

Kanungo, A.K., 2006. Excavation at Kopia 2006: a preliminary report. Puratattva 36, $111-120$.

Kanungo, A.K., 2010. Antiquity of glass in India: Excavations at Kopia. In: Tripathi, V., Upadhyay, P. (Eds.), Archaeology of the Ganga Basin, Paradigm Shift. vol. II. Sharada Publishing House, Delhi, pp. 451-476.

Kanungo, A.K., Misra, V.N., 2004. Excavation at Kopia: a preliminary report. Puratattva $34,116-126$

Kanungo, A.K., Shinde, V.S., 2005. Excavation at Kopia 2005: a preliminary report. Puratattva 35, 126-134

Kanungo, A.K., Misra, V.N., Dutta, K., Ravi Prasad, G.V., Yadava, M.G., Hodgins, G.W.L., 2010. Radiocarbon chronology of Kopia, an early glass manufacturing Centre in India. Archaeometry 52 (5), 899-918.

Labbé, A.J., 1985. Ban Chiang: Art and Prehistory of Northeast Thailand. Bowers Museum, Santa Ana, California.

Lal, B.B., 1952. Examination of some ancient glass specimens. Ancient India 8, 17-27.

Lamb, A., 1965. A note on glass fragments from Pengkalan Bujang, Malaya. J. Glass Stud. 7, 35-40.

Lankton, J.W., Dussubieux, L., 2006. Early glass in Asian maritime trade: a review and an interpretation of compositional analyses. J. Glass Stud. 48, 121-144.

Lankton, J., Dussubieux, L., 2013. Ancient glass from Southeast Asia. In: Janssens, K. (Ed.), Modern Methods for Analysing Archaeological and Historical Glass. John Wiley \& Sons, pp. 413-441.

Lankton, J., Dussubieux, L., Gratuze, B., 2008. Glass from Khao Sam Kaeo: transferred technology for an early Southeast Asian exchange network. In: Recent Advances in the Archaeology of Exchange in the Upper Thai-Malay Peninsula. 93. B. Ecole Française d'Extrême-Orient 2006, pp. 317-351.

Li, Feng, 1999. Elemental and Isotopic Analysis of Ancient Chinese Glass. Master Dissertation. University of Hong Kong.

Malleret, L., 1962. L'Archéologie du delta du Mékong. Tome Troisième, la culture du FouNan. Publications de l'Ecole Française d'Extrême-Orient, Paris.

Manguin, P.-Y., 2002. The amorphous nature of coastal polities in Insular Southeast Asia: restricted centres, extended peripheries. Mousson 5, 73-99.

Pearce, N.J.G., Perkins, W.T., Westgate, J.A., Gorton, M.T., Jackson, S.E., Neal, C.R., Chenery, S.P., 1997. A compilation of new and published major and trace element data for NIST SRM 610 and SRM 612 glass reference materials. Geostand. Newslett. 
XXI, 114-115.

Pelegrin, J., 2000. Technique et méthodes de taille pratiquées à Cambay. In: Roux, V. (Ed.), Cornaline de l'Inde. Des pratiques techniques de Cambay aux techno-système de l'Indus. Maison des Sciences de l'Homme, Paris, pp. 53-93.

Pryce, T.O., Bellina, B., 2017. High-tin bronze bowls and copper drums: non-ferrous archaeometallurgical evidence for Khao Sek's involvement and role in regional later 1st millennium BC Thai-Malay Peninsula exchange systems. Archaeol. Res. Asia (this issue).

Pryce, T.O., Murillo-Barroso, M., Biggs, L., Martinón-Torres, M., Bellina, B., 2017. The metallurgical industries. In: Bellina, B. (Ed.), Khao Sam Kaeo: a late prehistoric early city between the Indian Ocean and the South China Sea. Ecole française d'ExtrêmeOrient, Paris, pp. 500-545.

Qishan, Huang, 1996. Discovery and Research of Ancient Glass Utensils in Guangxi. International Congress on Glass, Beijing, pp. 481-485

Reinecke, A., Chieu, Nguyen, Dung, Lam Thi My, 2002. Neue Entdeckungen zur Sa Huynh-Kultur. Köln: Lindensoft, AVA-Forschungen 7.

Salisbury, A., Glover, I., 1997. New analyses of early glass from Thailand and Vietnam. Bead study trust. Newsletter 30, 7-14.
Singh, R.N., 1989. Ancient Indian Glass: Archaeology and Technology. Parimal Publications, Delhi (235 p).

Solheim, W.G., 2006. Archaeology and Culture in Southeast Asia: Unraveling the Nusantao. The University of the Philippines Press, Quezon City.

Subramanian, R., 1950. Analysis of ancient glass beads. Curr. Sci. India 19, 19-20.

an Thang, Dang, Hien, Vu Quoc, 1997. Excavation at Giong Ca Vo Site, Can Gio District, Ho Chi Minh City. J. Southeast Asian Archaeol. 30-45.

Varshney, Y.P., 1950. Glass in ancient India. The. Glass Ind. 31 (12), 632-634.

Varshneya, A.K., Tong, S.S.C., Gogte, V., 1988. Analysis of early glass objects from Nevasa excavations in India. Trans. Indian Ceram. Soc. 47 (7), 149-157.

White, J.C., 1982, Discovery of a Lost Bronze Age, The University Museum, the University of $\mathrm{f}$ Pennsylvania and the Smithsonian Institution.

Zhang, B., Li, Y.H., Ma, B., Gan, F.X., Zhang, Z.Q., Cheng, H.S., Yang, F.J., 2004. Nondestructive analysis of early glass unearthed in south China by external-beam PIXE. J. Radioanal. Nucl. Chem. 261 (2), 387-392.

Zhang, B., Cheng, H.S., Ma, B., Li, Q.H., Zhang, P., Gan, F.X., Yang, F.J., 2005. PIXE and ICP-AES analysis of early glass unearth from Xinjiang (China). Nucl. Inst. Methods Phys. Res. B 240, 559-564. 